\title{
Obstructed uterine venous return: a preventable cause of dysfunctional labor?
}

\author{
Thomas L Archer \\ Department of Anesthesiology, UT Health San Antonio, San Antonio, Texas, USA \\ Correspondence should be addressed to T L Archer; Email: tarcher0727@hotmail.com
}

\begin{abstract}
Dysfunctional labor is a common cause of cesarean delivery and may be caused by myometrial hypoxia. Obstruction of uterine venous return due to compression of the inferior vena cava by the gravid uterus or the abdominal wall may be an auxiliary cause of myometrial hypoxia which aggravates other causes.

Reproduction (2021) 161 V15-V17
\end{abstract}

Dysfunctional labor is a common cause of cesarean delivery (CD), particularly in obese parturients, and uterine atony is a closely-related failure of the myometrium to do its job properly. Dysfunctional labor and uterine atony undoubtedly have many causes, but Wray et al. have recently published an excellent review (Wray et al. 2021) in Reproduction entitled 'Hypoxia and Labour,' which presents abundant evidence from both rats and humans that myometrial hypoxia contributes to the pathogenesis of dysfunctional labor, and this hypothesis seems highly plausible given the need of working muscles for oxygen.

But what causes the myometrial hypoxia? The question is complicated since the dynamic oxygen economy of the myometrium - like that of the heart-should be examined in terms of both supply and demand, and hypoxia implies that demand outstrips supply at some point during pregnancy. Protracted maternal hypotension and sleep-disordered breathing in all patients, and endothelial dysfunction in obese patients, all limit the fetal and myometrial oxygen supplies (Prendergast 2020). Myometrial oxygen demand increases both with the frequency of uterine contractions but also with increasing uterine size, since Laplace's Law states that a larger muscular organ must generate a greater wall tension than a smaller organ in order to generate a given intracavitary pressure - and greater wall tension requires greater oxygen consumption. But obstructed uterine venous return, with resultant elevations in uterine venous pressure and reduction in uterine perfusion pressure (arterial minus venous pressure) always stands ready to exacerbate any or all of these other underlying causes of myometrial hypoxia. In addition to slowing down uterine perfusion, increased uterine venous pressure also causes uterine congestion and edema, which further reduces cellular oxygenation by increasing the diffusion distance for oxygen from capillaries to cells.
For decades 'aortocaval compression' by the gravid uterus has been known to cause both maternal and fetal distress (Couper et al. 2021), and the higher incidence of dysfunctional labor in obese patients may be explained by the fact that a heavy abdominal wall exacerbates any inferior vena cava (IVC) obstruction due to the uterus itself (Sawchuck \&Wittmann 2014). Little consideration appears to have been given, however, to the possible effects of such obstruction on the myometrium itself, independent of its effects on fetal oxygenation, and avoiding aortocaval compression during labor is often regarded simply as a way of maintaining maternal arterial blood pressure and thereby avoiding fetal distress. The venous side of the picture seems to be ignored and no thought seems to be given to the possibility of 'myometrial distress' as distinct from 'fetal distress'. The assumption seems to be that if we tip the patient 15-30 degrees to the left during labor, keep the maternal blood pressure up despite the effects of the epidural and don't see late decelerations of the fetal heart rate (FHR) following uterine contractions, then we have done our job and all is well. The implicit assumption seems to be that the uncomplaining but possibly hypoxic myometrium can take care of itself, as long as FHR and variability are acceptable.

If the myometrium does begin to contract poorly during labor, we attempt to stimulate it with oxytocin and measure its resulting performance using intrauterine pressure catheters, but perhaps what the uterus sometimes needs during labor is not more oxytocin but more oxygen (Archer 2020). Better myometrial performance might sometimes be obtained by correcting unrecognized IVC obstruction instead of administering oxytocin to a hypoxic myometrium. This not to deny that obesity may impair myometrial contractility via dietary and structural mechanisms that have nothing to do with obstruction to uterine venous return (Muir et al. 2016). 
With respect to the separability of fetal hypoxia from myometrial hypoxia, I would make the following observations: two distinct clinical syndromes are common: (1) the hypoxic fetus becomes bradycardic due to myometrial hyperactivity and (2) the uterus stops working even though the FHR and variability remain acceptable. A further ironic example of this discrepancy between the oxygen needs of the fetus and those of the myometrium is that when the hypoxia-distressed myometrium stops contracting, fetal distress usually resolves (Archer 2020, Wray et al. 2021)! In other words, the very process of dysfunctional labor may protect the fetus from hypoxic injury. A final hint as to how the fetoplacental unit and its underlying basal myometrium may 'compete' with the parietal myometrium for scarce perfusion is provided by the myometrial arterio-venous (AV) shunts that have been discovered within the basal myometrium underlying the placenta (Schaaps et al. 2005). Increased uterine venous pressure or other factors during labor may enlarge these shunts and allow the diversion of blood away from the hard-working and needful parietal myometrium. (See Fig. 1 for a graphical depiction of this 'myometrial steal' proposal.) A recent MRI study (Couper et al. 2021) of maternal and fetal perfusion of the placenta and its underlying myometrium in non-laboring women found that both decreased in the supine position as compared to the left lateral decubitus position, however, this study did not address the issue of positional parietal myometrial perfusion during labor.

Hence, we have three distinct 'players' whose differing oxygen supplies must be protected simultaneously and independently: the mother, the fetus, and the myometrium itself! But in the absence of fetal distress, how can we know if the myometrium is independently hypoxic? It is not practical to make such a direct determination at present, but we can minimize any aggravating role that obstructed uterine venous return may be playing by using maternal cardiac output (CO) as a general purpose marker for IVC obstruction.

A common factor in position-related fetal and myometrial hypoxia would be positional obstruction of the IVC resulting in reduced venous return. Since venous return equals $\mathrm{CO}$ in a steady state, reductions of maternal CO during labor would then serve as an 'early warning system' for IVC obstruction, allowing correction of the problem by patient repositioning before such obstruction could result in either fetal distress or dysfunctional labor due to myometrial hypoxia. By keeping the maternal $\mathrm{CO}$ at its positional maximum we would stand a good chance of minimizing any IVC obstruction, and thereby keep uterine venous pressure low and both the fetoplacental unit and the myometrium well-perfused.

But how can we follow maternal cardiac output during labor? For decades cardiac output has been difficult to measure, requiring invasive, dangerous, time-consuming and intermittent techniques, such as pulmonary artery catheterization. Even now, the reliability of the absolute values obtained from non-invasive and continuous 'measurement' of CO is doubtful. But it is increasingly recognized that electrical cardiometry or bioreactance allows the safe, continuous and clinically meaningful trending of $\mathrm{CO}$ within individual patients over time (Liu et al. 2015, Feng \& Liu 2020), and all that is required to successfully maximize cardiac output is the ability to record it reliably.

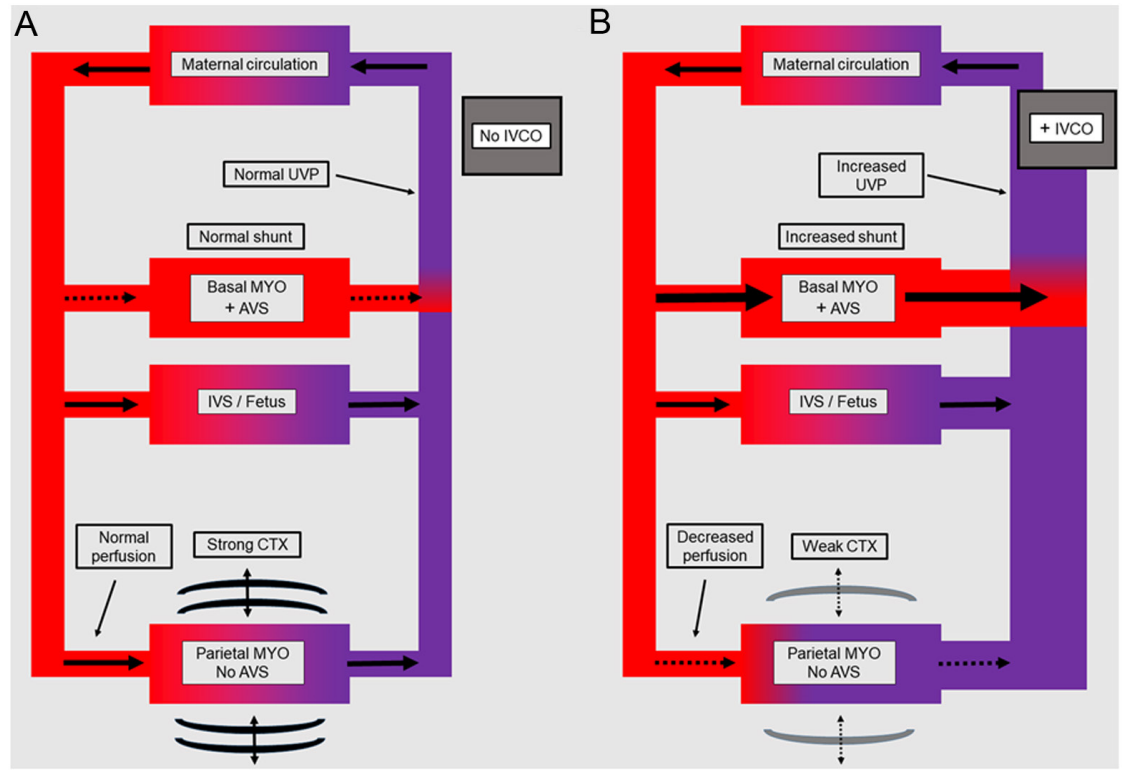

Reproduction (2021) 161 V15-V17
Figure 1 A speculative three compartment model for uterine circulation in healthy labor (A) vs dysfunctional labor (B). The three compartments are: (1) intervillous spaces, (2) basal myometrial AV shunts underlying placenta, (3) parietal myometrium (contracting uterine muscle separate from placenta). (A) In normal labor, the uterine venous pressure is low and perfusion of both the intervillous spaces and the parietal myometrium is brisk. Contractions are strong. The basal myometrial AV shunts regulate and optimize intervillous pressure and flow. (B) In dysfunctional labor, the uterine venous pressure is high and flow through basal myometrial AV shunts increases, stealing perfusion from the hard-working parietal myometrium. Contractions weaken due to hypoxia, acidosis and edema. Perhaps the system defends adequate perfusion of the intervillous spaces to ensure fetal survival. + AVS, arteriovenous shunts present; No AVS, arteriovenous shunts absent; CTX, contractions; IVCO, inferior vena cava obstruction; IVS, intervillous spaces; MYO, myometrium; UVP, uterine venous pressure. 
Certainly, the combined hemodynamics of pregnancy and obesity are exceedingly complex (Vinayagam et al. 2017), involving vasodilation, sympathetic activation of the renin-angiotensin-aldosterone system and changes in endothelial function. But loaded on top of this neuroendocrine complexity are the relatively simple and reversible hemodynamic effects of mechanical compression of veins by the abdominal wall or uterus itself. The clinically important question I would like to answer is straightforward: Can unblocking uterine venous drainage by appropriate patient positioning improve outcomes?

The study would compare outcomes in two groups of obese patients. Group 1 would receive routine care despite the acquisition of cardiac output data, whereas Group 2 would be positioned to maximize maternal cardiac output, as long as the FHR remained acceptable. Of course, one would control for all the other factors influencing labor progress, summarized as 'the powers, the passage and the passenger'.

If cardiac output-guided maternal positioning were shown to reduce the incidence of $C D$ for dysfunctional labor or postpartum uterine atony, a new labor monitoring routine might be introduced, as follows: once the patient was comfortable with her epidural, she would be positioned so as to maximize her CO, even if the FHR were also favorable in other positions. By maintaining unobstructed uterine venous return and optimal overall uterine perfusion, this approach might protect the separate interests of both fetus and myometrium.

One further point about patient mobility during labor: as an obstetric anesthesiologist I am proud of the role that epidural analgesia plays in reducing the pain of childbirth, but - teleologically speaking - painful labor has the 'advantage' to the fetus of keeping the parturient in constant motion, thereby preventing the uterus from obstructing its own venous return. If labor were painless, the parturient might lie serenely in a position in which her fetus or her myometrium might not get all the oxygen they need. If CO-guided maternal positioning protected the oxygen supplies of both fetus and myometrium, we might have the best situation of all $-\mathrm{a}$ comfortable and rested patient, a well oxygenated fetus and a hard-working, 'happy' myometrium that contracts appropriately both during and after labor and delivery.

\section{Declaration of interest}

The author declares that there is no conflict of interest that could be perceived as prejudicing the impartiality of this article.

\section{Funding}

The author did not receive any specific grant from any funding agency in the public, commercial or not-for-profit sector for writing this articl.

\section{References}

Archer TL 2020 Cardiac output-guided resuscitation of the uterus: an obese patient has dysfunctional labor which resolves with position change. Coincidence or possible therapy? In Obstetric Anesthesia: A CaseBased and Visual Approach, 1st ed., Ch 32, pp. 293-301. Ed TL Archer. Switzerland: Springer. (https://doi.org/10.1007/978-3-030-26478-9_32)

Couper S, Clark A, Thompson JMD, Flouri D, Aughwane R, David AL, Melbourne A, Mirjalili A \& Stone PR 2021 The effects of maternal position, in late gestation pregnancy, on placental blood flow and oxygenation: an MRI study. Journal of Physiology 599 1901-1915. (https://doi.org/10.1113/JP280569)

Feng SM \& Liu J 2020 Electrical velocimetry has limited accuracy and precision and moderate trending ability compared with transthoracic echocardiography for cardiac output measurement during cesarean delivery: a prospective observational study. Medicine 99 e21914. (https://doi.org/10.1097/MD.0000000000021914)

Liu Y, Pian-Smith MC, Leffert LR, Minehart RD, Torri A, Coté C, Kacmarek RM \& Jiang Y 2015 Continuous measurement of cardiac output with the electrical velocimetry method in patients under spinal anesthesia for cesarean delivery. Journal of Clinical Monitoring and Computing 29 627-634. (https://doi.org/10.1007/s10877-0149645-8)

Muir R, Ballan J, Clifford B, McMullen S, Khan R, Shmygol A, Quenby S \& Elmes M 2016 Modelling maternal obesity: the effects of a chronic highfat, high-cholesterol diet on uterine expression of contractile-associated proteins and ex vivo contractile activity during labour in the rat. Clinical Science 130 183-192. (https://doi.org/10.1042/CS20150539)

Prendergast C 2020 Maternal phenotype: how do age, obesity and diabetes affect myometrial function? Current Opinion in Physiology 13 108-116. (https://doi.org/10.1016/j.cophys.2019.10.016)

Sawchuck DJ \& Wittmann BK 2014 Pre-eclampsia renamed and reframed: intra-abdominal hypertension in pregnancy. Medical Hypotheses $\mathbf{8 3}$ 619-632. (https://doi.org/10.1016/j.mehy.2014.08.001)

Schaaps JP, Tsatsaris V, Goffin F, Brichant JF, Delbecque K, Tebache M, Collignon L, Retz MC \& Foidart JM 2005 Shunting the intervillous space: new concepts in human uteroplacental vascularization. American Journal of Obstetrics andGynecology 192 323-332. (https:// doi.org/10.1016/j.ajog.2004.06.066)

Vinayagam D, Gutierrez J, Binder J, Mantovani E, Thilaganathan B \& Khalil A 2017 Impaired maternal hemodynamics in morbidly obese women: a case-control study. Ultrasound in Obstetrics and Gynecology 50 761-765. (https://doi.org/10.1002/uog.17428)

Wray S, Alruwaili M \& Prendergast C 2021 Hypoxia and reproductive health: Hypoxia and labour. Reproduction 161 F67-F80. (https://doi. org/10.1530/REP-20-0327)

Received 6 January 2021

First decision 25 January 2021

Revised Manuscript received 16 February 2021

Accepted 22 February 2021 